\title{
Éditorial
}

\section{Un numéro différent}

Dès sa réception vous avez remarqué que ce numéro était différent. Il est plus épais, au lieu de 4 articles dans le $n^{\circ} 3$ de 2006 qui était notre numéro du $40^{\mathrm{e}}$ anniversaire, nous en avons 8. Que s'est il passé ?

La réponse est simple, le conseil d'administration et les sections ont répondu à l'appel du comité de rédaction. Les manifestations de la SFRP font très souvent l'objet de présentations de très bonne qualité mais leurs auteurs, souvent, s'arrêtaient là. En accord avec le conseil d'administration, nous avons demandé aux organisateurs de ces journées de sensibiliser ces conférenciers dès l'organisation de leur manifestation, au besoin de rendre pérenne leur effort en publiant dans notre journal, celui de la SFRP.

Nous sommes heureux d'enregistrer les premiers effets.

Les sessions tutoriales du congrès de Reims recèlent d'excellentes synthèses, utiles à l'ensemble de la profession, comme leur succès en atteste. Vous trouverez dans nos colonnes celles que le comité de rédaction a sélectionnées avec sa rigueur habituelle. Il me plait de noter que, bien qu'il n'y ait eu aucune concertation, ce numéro publie un article de Catherine Mercat-Rommens, récompensée par ailleurs à Reims (prix « poster»). Ceci devrait encourager les deux autres lauréats à nous envoyer leur contribution: Bouchra Habib et l'équipe de Saclay associant le code Monte Carlo Pénélope à la radiothérapie et Mfoihaya Bédja qui se préoccupent de l'exposition de la population française au champ magnétique $50 \mathrm{~Hz}$. Nous les félicitons tous.

Pour les prochains numéros, la section de protection technique a réussi à mobiliser certains auteurs des journées « Codes de calcul ». Vous en verrez bientôt le résultat.

Ainsi, nous pensons que notre journal devient de plus en plus le journal d'une profession, la radioprotection. Il reflète, je l'espère, la radioprotection au quotidien, avec une seule exigence : la qualité. Mais ces nouveaux auteurs ne doivent pas démobiliser nos auteurs plus traditionnels.

Alors, continuons nos efforts et merci à tous, les auteurs en tout premier, les évaluateurs, les « chasseurs d'articles » et, bien entendu notre comité, sans oublier un conseil d'administration tout acquis à notre cause.

Le comité de rédaction a tenu en juin sa réunion annuelle délocalisée à SochauxMontbéliard chez notre ami Libor Mackovika ; une journée fort sympathique.

Henri Métivier

Président du comité de la revue

DOI: 10.1051/radiopro:2007038

RADIOPROTECTION - VOL. 42 - N 3 (2007)

Article published by EDP Sciences and available at http://www.edpsciences.org/radiopro or http://dx.doi.org/10.1051/radiopro:2007038 